\title{
AN ABSTRACT NONLINEAR CAUCHY-KOVALEVSKA THEOREM $\left({ }^{1}\right)$
}

\author{
BY \\ FRANCOIS TREVES
}

\begin{abstract}
A nonlinear version of Ovcyannikov's theorem is proved. If $F(u, t)$ is an analytic function of $t$ real or complex and of $u$ varying in a scale of Banach spaces, valued in a scale of Banach spaces, the Cauchy problem $u_{t}=F(u, t), u(0)=u_{0}$, has a unique analytic solution. This is an abstract version of the Cauchy-Kovalevska theorem which can be applied to equations other than partial-differential, e.g. to certain differential-convolution or, more generally, differential-pseudodifferential equations.
\end{abstract}

Introduction. This is a continuation of recent work revolving around the, so-called, Ovcyannikov's theorem (see [T1], [T2], [S-T1]; see also [O1]). This time we study a nonlinear extension of that result. The extension concerns Cauchy problems of the kind

$$
d x / d t=F(x, t), \quad x(0)=x_{0},
$$

where the solution $x=x(t)$ is sought in the class of analytic functions of the (complex) variable $t$ valued in a scale of Banach spaces $\left\{X_{s}\right\}$ and where $F(x, t)$ is analytic with respect to both $x$ and $t$. The type of analyticity of $F$ with respect to $x$ in which we are here interested is related to the basic condition in Ovcyannikov's theorem, namely that for suitable constants $r>0, C>0$ and for all $p=0,1, \ldots$, the $p$ th Fréchet derivative of $F$ with respect to $x$, at the point $x_{0}$ of $X_{1}$ (the smallest of the spaces $X_{s}$ ), is a $p$-linear mapping of $X_{s}^{p}$ into $X_{s^{\prime}}$ whenever $0 \leqq s^{\prime}<s \leqq 1$, with norm

$$
\leqq \frac{C}{s-s^{\prime}} r^{-p} p !
$$

(and depends holomorphically on $t$ in a disk centered at $t=0$ ). Under these sole conditions the desired existence-and-uniqueness theorem is proved. We have thought worthwhile publishing this theorem because of its neatness, and also because its proof is not a mere extension of the proof of the linear Ovcyannikov theorem. (The induction estimate in the iteration procedure must be slightly modified: see $(2.2)_{n}$.)

Received by the editors July 3, 1969.

AMS Subject Classifications. 3503, 3536, 3537.

Key Words and Phrases. Cauchy-Kovalevska, nonlinear, Cauchy problem, scale of Banach spaces, analytic, Ovcyannikov, Banach algebras, Fourier transform, analytic functionals, Gevrey class.

(1) This work has been done in part under NSF grant GP-07346. 
As for the applications of the theorem, we describe rapidly some examples in $\$ \S 5$ and 6 (after having introduced a few "concrete" scales of Banach algebras, obviously relevant in the present context, in \$4). No doubt that many of the results obtained by applying the abstract theorem are known. But this does not seem to be the case for some of them, and it is hard so see how to reach them by a different procedure than the one presented here. An example of these concerns the Cauchy problems of the kind:

$$
\begin{gathered}
\partial^{m} u / \partial t^{m}=f\left(A^{0} u, A^{1} \partial u / \partial t, \ldots, A^{m-1} \partial^{m-1} u / \partial t^{m-1}, x, t\right), \\
\partial^{j} u /\left.\partial t^{j}\right|_{t=0}=u^{j}(x), \quad 0 \leqq j<m,
\end{gathered}
$$

where the $A^{j}$ are certain kinds of (linear) convolution operators (or even of "pseudodifferential" operators) of a suitable degree (see \$6). Here the basic Banach space scales consist of Gevrey functions in open subsets of $R^{N}$ (where the variable is denoted by $x$ ). We may take $f$ to be an analytic function of all its arguments with the exception of $x$, in which it is allowed to be Gevrey (of an appropriate order and type; this applies also to the Cauchy data).

The program of study begun in [T1], developed in [T2], [S-T1] and in the present article, ought to be pushed further. Problems of the type $(*)$ where the analyticity of $F$ is relinquished, and replaced by other types of regularity (such, for instance, as Gevrey), must be studied. We hope to return to these questions in future publications.

We use the standard multi-index notation: if $p=\left(p_{1}, \ldots, p_{N}\right)$ is an $N$-tuple of nonnegative integers $p_{j}$, we write $|p|=p_{1}+\cdots+p_{N}, p !=p_{1} ! \cdots p_{N} !$,

$$
\left(\begin{array}{l}
p \\
q
\end{array}\right)=p ! /[(p-q) ! q !], \text { etc. }
$$

1. Statement of the theorem about single first order equations. We deal with a scale of Banach spaces $X_{s}(0 \leqq s \leqq 1)$, which means that if $s^{\prime} \leqq s, X_{s}$ is a linear subspace of $X_{s^{\prime}}$ and the injection $X_{s} \rightarrow X_{s^{\prime}}$ is continuous and has norm $\leqq 1$. The norm in $X_{s}$ will be denoted by \|\|$_{s}$.

We shall need a special notion of analyticity applicable to mappings defined on subsets of every space $X_{s}$. For each $p=1,2, \ldots$, let be given a $p$-linear map

$$
\begin{gathered}
p \text { times } \\
F_{p}: X_{s} \times \cdots \times X_{s} \rightarrow X_{s^{\prime}}
\end{gathered}
$$

where $s$ and $s^{\prime}$ is any pair of numbers such that $0 \leqq s^{\prime}<s \leqq 1$. Let us define also $F_{p}$ for $p=0$ as a fixed element of the intersection of the $X_{s}, s<1$. We suppose that there is a function $M\left(s, s^{\prime}\right)>0$, defined for $s^{\prime}<s$, and a number $r>0$ such that:

(1.1) $\left\|F_{0}\right\|_{s^{\prime}} \leqq M\left(1, s^{\prime}\right)\left(s^{\prime}<1\right)$;

(1.2) when $p=1,2, \ldots$, for all $p$-tuples $x_{1}, \ldots, x_{p}$ in $X_{s}$,

$$
\left\|F_{p}\left(x_{1}, \ldots, x_{p}\right)\right\|_{s^{\prime}} \leqq M\left(s, s^{\prime}\right) r^{-p}\left\|x_{1}\right\|_{s} \cdots\left\|x_{p}\right\|_{s} .
$$

Let us then set, for any $x$ in $X_{s}$, 
(1.3) $\hat{F}_{p}(x)=F_{p}(x, \ldots, x)$.

Let now $x_{0}$ be an arbitrary point of $X_{1}$, the "smallest" of the Banach spaces $X_{s}$. By virtue of (1.1) and (1.2), the series

$$
\sum_{p=0}^{+\infty} \hat{F}_{p}\left(x-x_{0}\right)
$$

converges in $X_{s^{\prime}}$ uniformly with respect to $x$ in every open ball

$$
\left\{x \in X_{s} ;\left\|x-x_{0}\right\|_{s}<r^{\prime}\right\}, \quad r^{\prime}<r .
$$

The sum of the series (1.4) defines therefore an analytic mapping of the open ball with radius $r$, centered at $x_{0}$, into $X_{s^{\prime}}$. Obviously, not every such analytic mapping can be represented by a series of the kind of (1.4) (under the assumptions (1.1), (1.2)). It is the ones that can be so represented that will be of interest to us. We shall refer to them as analytic functions on the Banach space scale $X_{s}$ in a neighborhood of $x_{0} \in X_{1}$.

Note that, for each fixed pair $s, s^{\prime}\left(s^{\prime}<s\right)$, the numbers $M\left(s, s^{\prime}\right)$ which can be used in (1.1), (1.2) have a minimum: this minimum defines a function of $s, s^{\prime}$ which we shall refer to as the module of the function (1.4).

We introduce now a complex variable $t$, varying in the disk $|t|<\eta(\eta>0)$. We shall then be interested in functions $F(x, t)$, analytic with respect to $t$ and also with respect to $x$-but on the Banach space scale $\left\{X_{s}\right\}$ and in a neighborhood of $x_{0}$. There are various equivalent ways of introducing such functions. The most convenient to us is probably to use a representation

$$
F(x, t)=\sum_{p=0}^{+\infty} \sum_{q=0}^{+\infty} \hat{F}_{p, q}\left(x-x_{0}\right) t^{q},
$$

where

(1.6) $\hat{F}_{0, q}=F_{0, q} \in X_{s^{\prime}}$ for all $s^{\prime}<1$,

(1.7) $\left\|F_{0, q}\right\|_{s^{\prime}} \leqq M\left(1, s^{\prime}\right) \eta^{-q}(q=0,1, \ldots)$,

and where, for $p=1,2, \ldots$, and for any $x$ in $X_{s}$,

(1.8) $\hat{F}_{p, q}(x)=F_{p, q}(x, \ldots, x)$,

where $F_{p, q}$ is a $p$-linear mapping $X_{s} \rightarrow X_{s^{\prime}}\left(0 \leqq s^{\prime}<s \leqq 1\right)$ satisfying (for a suitable constant $r>0$ and all $p$-tuples $x_{1}, \ldots, x_{p}$ in $X_{s}$ )

$$
\left\|F_{p, q}\left(x_{1}, \ldots, x_{p}\right)\right\|_{s^{\prime}} \leqq M\left(s, s^{\prime}\right) r^{-p} \eta^{-q}\left\|x_{1}\right\|_{s} \cdots\left\|x_{p}\right\|_{s} .
$$

The function $M\left(s, s^{\prime}\right)>0$ is the module of the mapping $F(x, t)$.

We may now concern ourselves with the Cauchy problem:

$$
d x / d t=F(x, t), \quad x(0)=x_{0} .
$$

We would like to state and prove an existence-and-uniqueness theorem for analytic solutions of (1.10). This is feasible provided that the singularity of the module 
function $M\left(s, s^{\prime}\right)$ at the diagonal $s=s^{\prime}$ is at worst a pole of degree one, more precisely, provided that

(1.11) there is a constant $C>0$ such that, for all $s, s^{\prime}, 0 \leqq s^{\prime}<s \leqq 1$,

$$
M\left(s, s^{\prime}\right) \leqq C /\left(s-s^{\prime}\right) .
$$

THEOREM 1.1. Suppose that the mapping $F(x, t)$ is given by (1.5) and that the assumptions (1.6), (1.7), (1.8), (1.9) are satisfied. Suppose furthermore that (1.11) holds.

Then there is a unique analytic solution $x(t)$ of (1.10) valued in the scale of Banach spaces $\left\{X_{s}\right\}$-in the following sense:

(Existence) There is a number $\delta, 0<\delta<\eta$, such that, given any $s, 0 \leqq s<1, x(t)$ is an analytic function of $t,|t|<\delta(1-s)$, with values in $X_{s}$.

(Uniqueness) If for some $s, 0 \leqq s \leqq 1$, there is an analytic function of $t$ in an open disk centered at $t=0$, valued in $X_{s}$ and satisfying (1.10), it must be equal to $x(t)$.

The proof of Theorem 1.1 will provide an estimate for the number $\delta$ and for the norm $\|x(t)\|_{s}$ (see Remark 2.1).

2. Proof of the Theorem 1.1. To begin with, we reason formally. Let us set $x-x_{0}=\sum_{n=1}^{+\infty} x_{n} t^{n}$. If we make use of (1.5), (1.6) and (1.8) we may write

$$
\begin{aligned}
F(x, t) & =\sum_{p=0}^{+\infty} \sum_{q=0}^{+\infty} \hat{F}_{p, q}\left(x_{n_{1}}, \ldots, x_{n_{p}}\right) t^{q+n_{1}+\cdots+n_{p}} \\
& =\sum_{k=0}^{+\infty} t^{k} \sum_{q+n_{1}+\cdots+n_{p}=k} \hat{F}_{p, q}\left(x_{n_{1}}, \ldots, x_{n_{p}}\right),
\end{aligned}
$$

where, in the last summation, $p$ is allowed to be any integer $\geqq 0$ (but in fact is compelled to be $\leqq k-q$ since the $n_{i}$ 's are all $>0$ ). Since, on the other hand, $x^{\prime}(t)=\sum_{n=1}^{+\infty} n x_{n} t^{n-1}$, we see that equations (1.10) are equivalent with the sequence of equations, for $k=0,1, \ldots$,

$$
x_{k+1}=\frac{1}{k+1} \sum_{q+n_{1}+\cdots+n_{p}=k} \hat{F}_{p, q}\left(x_{n_{1}}, \ldots, x_{n_{p}}\right) .
$$

This determines $x_{k+1}$ as a function of the $x_{n}$ for $n \leqq k$ and therefore proves the uniqueness of the solution. In order to prove its existence, we shall establish an estimate for the norm $\left\|x_{n}\right\|_{s}$. This estimate will be stated as an induction hypothesis, on $n=1,2, \ldots$ :

$$
\left\|x_{n}\right\|_{s} \leqq\left(r / 8 S n^{2}\right)(A /(1-s))^{n},
$$

where $S$ is the sum of the series $\sum_{p=1}^{+\infty} 1 / p^{2}$ and where $A$ is a large number, independent of $s$ and $n$, to be chosen later.

Let us first note that $(2.2)_{1}$ is true, provided that $A$ is sufficiently large. Indeed, according to (2.1), $x_{1}=\hat{F}_{0,0}=F_{0,0}$, hence, by (1.7) and (1.11), $\left\|x_{1}\right\|_{s} \leqq C /(1-s)$, and it suffices to require $A \geqq 8 r^{-1} S C$. 
We suppose heretofore that $(2.2)_{n}$ holds for all $n \leqq k$. We derive from (1.7) and (1.9), by way of (2.1),

$$
\left\|x_{k+1}\right\|_{s} \leqq \frac{C}{k+1} \sum_{q=0}^{k} \eta^{-q} \sum_{|n|=k-q} \frac{r^{-p}}{\varepsilon_{q}}\left\|x_{n_{1}}\right\|_{s+\varepsilon_{q}} \cdots\left\|x_{n_{p}}\right\|_{s+\varepsilon_{q}},
$$

where $|n|=n_{1}+\cdots+n_{p}$ and $\varepsilon_{q}=(1-s) /(k-q+1)$. By the induction hypothesis,

$$
\begin{aligned}
\left\|x_{n_{1}}\right\|_{s+\varepsilon_{q}} \cdots\left\|x_{n_{p}}\right\|_{s+\varepsilon_{q}} & \leqq \frac{r^{p}}{(8 S)^{p}}\left(\frac{A}{1-S-\varepsilon_{q}}\right)^{k-q}\left(n_{1} \cdots n_{p}\right)^{-2} \\
& =\frac{r^{p}}{(8 S)^{p}}\left(1+\frac{1}{k-q}\right)^{k-q}\left(\frac{A}{1-S}\right)^{k-q}\left(n_{1} \cdots n_{p}\right)^{-2},
\end{aligned}
$$

whence

$$
\left\|x_{k+1}\right\|_{s} \leqq \frac{1}{k+1} \frac{C e}{1-s} \sum_{q=0}^{k}(k-q+1) \eta^{-q}\left(\frac{A}{1-s}\right)^{k-q} B_{k-q},
$$

where $B_{i}=\sum_{|n|=i}(8 S)^{-p}\left(n_{1} \cdots n_{p}\right)^{-2}\left(B_{0}=1\right)$.

We require $A \geqq 2 / \eta$, whence, by (2.3),

$$
\left\|x_{k+1}\right\|_{s} \leqq \frac{1}{k+1} \frac{C e A^{k}}{(1-s)^{k+1}} \sum_{q=0}^{k}(k-q+1) 2^{-q} B_{k-q} .
$$

In view of (2.4), $(2.2)_{k+1}$ will follow from the two lemmas which we state now and prove later.

LEMMA 2.1. Let $p$ be any integer $>0$. Then, for all integers $i \geqq p$,

$$
i^{2} \sum_{|n|=i}\left(n_{1} \cdots n_{p}\right)^{-2} \leqq(4 S)^{p-1}
$$

LeMmA 2.2. We have, for all $m=1,2, \ldots, m 2^{-m} \sum_{n=1}^{m} 2^{n} / n \leqq 4$.

Let us show how these lemmas imply $(2.2)_{k+1}$ and, by way of consequence, Theorem 1.1.

First we apply Lemma 2.1. Thus we see that (for $i=1,2, \ldots$ )

$$
B_{i} \leqq \frac{1}{8 S i^{2}} \sum_{p=1}^{+\infty} 2^{-p-1}=\frac{1}{4 S i^{2}} \leqq \frac{1}{S(i+1)^{2}},
$$

using the fact that $(i+1)^{2} \leqq 4 i^{2}$ if $i \geqq 1$. By (2.4) this leads to

$$
\left\|x_{k+1}\right\|_{s} \leqq \frac{1}{k+1} \frac{1}{S} \frac{C e A^{k}}{(1-s)^{k+1}} \sum_{q=0}^{k}(k-q+1)^{-1} 2^{-q} .
$$

Lastly, we apply Lemma 2.2 , observing that

$$
\sum_{q=0}^{k}(k-q+1)^{-1} 2^{-q}=2^{k+1} \sum_{r=1}^{k+1} 2^{r} / r \leqq 4 /(k+1),
$$

whence $(2.2)_{k+1}$ if we require that $A \geqq 32 r^{-1} S C$. 
Proof of Lemma 2.1. Induction on $p$. The statement is trivial if $p=1$. We may write, for arbitrary $p>1$,

$$
\sum_{|n|=i} \frac{i^{2}}{\left(n_{1} \cdots n_{p}\right)^{2}}=\sum_{n_{1}+j=i}\left(\frac{i}{n_{1} j}\right)^{2} \sum_{n_{2}+\cdots+n_{p}=j}\left(\frac{j}{n_{2} \cdots n_{p}}\right)^{2} .
$$

The right-hand side is, by the induction hypothesis on $p$,

$$
\leqq(4 S)^{p-2} \sum_{n_{1}+j=i}\left(\frac{i}{n_{1} j}\right)^{2}
$$

and everything reduces to proving the result when $p=2$. Now, $i / n(i-n)=1 / n$ $+1 /(i-n)$, hence

$$
\sum_{n=1}^{i-1}\left\{\frac{i}{n(i-n)}\right\}^{2} \leqq 2 \sum_{n=1}^{i-1}\left(\frac{1}{n^{2}}+\frac{1}{(i-n)^{2}}\right)=4 \sum_{n=1}^{i-1} \frac{1}{n^{2}} \leqq 4 S .
$$

Proof of Lemma 2.2. Let us set $S_{m}=m 2^{-m} \sum_{n=1}^{m} 2^{n} / n$. We have $S_{m}=1+$ $\frac{1}{2}(m /(m-1)) S_{m-1}$. If $m>2$, we have $m /(m-1) \leqq 3 / 2$, therefore $S_{m} \leqq 1+(3 / 4) S_{m-1}$. This shows that if $S_{m-1} \leqq 4$, then also $S_{m} \leqq 4$. But $S_{1}=1$ and $S_{2}=2$.

REMARK 2.1. The proof of Theorem 1.1, precisely the inequalities $(2.2)_{n}$, provide an estimate for the number $\delta$ and for the norm $\|x(t)\|_{s}(0 \leqq s<1)$. We have

$$
\left\|x(t)-x_{0}\right\|_{s} \leqq \frac{r}{8 S} \sum_{n=1}^{+\infty} \frac{1}{n^{2}}\left(\frac{A|t|}{1-s}\right)^{n}
$$

and therefore, the series $x(t)=\sum_{n=0}^{+\infty} x_{n} t^{n}$ converges uniformly in $X_{s}$ when $t$ remains in the closed disk $|t| \leqq(1-s) / A$; thus we may take $\delta=1 / A$. For such $t$ 's,

$$
\left\|x(t)-x_{0}\right\|_{s} \leqq(r / 8)|t| /(1-s) .
$$

3. Systems of differential equations. In this section we look at first order systems of the kind

(3.1) $d x^{i} / d t=F^{i}\left(x^{1}, \ldots, x^{m}, t\right), i=1, \ldots, m$,

with prescribed initial conditions

(3.2) $x^{i}(0)=x_{0}^{i}, i=1, \ldots, m$.

Each variable $x^{i}$ will vary in a Banach space scale $X_{s}^{i}(0 \leqq s \leqq 1)$ and the initial data $x_{0}^{i}$ will belong to $X_{1}^{i}(i=1, \ldots, m)$. For each $i$, we shall have a series representation

$$
F^{1}\left(x^{1}, \ldots, x^{m}, t\right)=\sum_{p_{1}=0}^{+\infty} \ldots \sum_{p_{m}=0}^{+\infty} \sum_{q=0}^{+\infty} \hat{F}_{p, q}^{i}\left(x^{1}-x_{0}^{1}, \ldots, x^{m}-x_{0}^{m}\right) t^{q},
$$

where we have used the multi-indices notation $p=\left(p_{1}, \ldots, p_{m}\right)$. We have

$$
\hat{F}_{p, q}^{i}\left(x^{1}, \ldots, x^{m}\right)=F_{p, q}^{i}\left(x^{1}, \ldots, x^{1} ; \ldots ; x^{m}, \ldots, x^{m}\right),
$$

where $F_{p, q}$ is a continuous $p_{j}$-linear mapping in the $x^{j}$-variable, from any $X_{s}^{j}$ into any $X_{z^{\prime}}^{i}$ if $0 \leqq s^{\prime}<s \leqq 1$. 
As before, we shall impose a certain upper bound on the norm of the multilinear mapping $F_{p, q}^{i}: X_{s}^{1} \times \cdots \times X_{s}^{m} \rightarrow X_{s^{\prime}}^{i}$, depending on $p, q, s$ and $s^{\prime}$-but also on $i$. The norm of $F_{p, q}^{i}$ is given by

$$
\left\|F_{p, q}^{i}\right\|_{s, s^{\prime}}=\sup \left\|F_{p, q}^{i}\left(x_{1}^{1}, \ldots, x_{p_{1}}^{1} ; \ldots ; x_{1}^{m}, \ldots, x_{p_{m}}^{m}\right)\right\|_{s^{\prime}},
$$

where the supremum is taken when every $x_{j}^{i}\left(1 \leqq j \leqq p_{i}\right)$ ranges over the unit sphere of $X_{s}^{i}(i=1, \ldots, m)$.

We shall assume that there are $m$ integers $d_{i} \geqq 0(i=1, \ldots, m)$ such that, for each $i$,

$$
\left\|F_{p, q}^{i}\right\|_{s, s^{\prime}} \leqq M\left(s, s^{\prime}\right)^{d_{i} r^{-|p|} \eta^{-q}} .
$$

We seek an existence-and-uniqueness theorem for analytic solutions to (3.1)-(3.2). To obtain it, it is not enough to assume that (1.11) holds, as shown in the case where $m=1, d_{1}=2$. We shall have to make further assumptions on the system (3.1). That the right assumptions can be found, under which the desired existenceand-uniqueness of solutions can be proved, is evident on examples such as the following one:

$$
d x^{1} / d t=F^{1}\left(x^{2}\right), \quad d x^{2} / d t=0,
$$

in which $d^{1}$ can be any integer $>0$. As a matter of fact, it is not difficult to state what these "right" assumptions are.

For each pair $i, j, 1 \leqq i, j \leqq m$, we introduce a number $\theta_{j}^{i}$ equal to zero if $F^{i}\left(x^{1}, \ldots, x^{m}, t\right)$ is independent of $x^{j}$, to one otherwise. Consider then the polynomial in two indeterminates $\lambda, \mu$,

$$
\operatorname{det}\left(\lambda I-\left(\mu^{d_{i}} \theta_{j}^{i} c_{j}^{i}\right)\right) \text {, }
$$

where $c_{j}^{i}$ are $m^{2}$ complex numbers (and $I$ stands for the $m \times m$ identity matrix). Our assumption will be:

(3.5) Whatever the complex numbers $c_{j}^{i}$, the degree of (3.4) with respect to $\lambda, \mu$ is $\leqq m$.

The motivation for this condition can be seen as follows. Let $z$ be a complex variable and consider the system of linear partial differential equations

$$
\frac{\partial u^{i}}{\partial t}=\sum_{j=1}^{m} \theta_{j}^{i} c_{j}^{i}\left(\frac{\partial}{\partial z}\right)^{d_{i}} u^{j}, \quad i=1, \ldots, m .
$$

If condition (3.4) is satisfied, the Cauchy-Kovalevska theorem applies to it (with Cauchy data prescribed at $t=0$ ).

We may then state

THEOREM 3.1. Suppose that the preceding hypotheses, in particular (3.3) and (3.5) are satisfied. Suppose moreover that (1.11) holds.

Then we have the same conclusion as in Theorem 1.1, this time valid for the Cauchy problem (3.1)-(3.2) instead of (1.10). 
The proof of Theorem 3.1 is essentially similar to the one of Theorem 1.1, although somewhat more cumbersome (cf. also the standard proof of the CauchyKovalevska theorem using majorant series).

In the application, most higher order equations or, for that matter, most systems of such equations, can be transformed into equations of the type (3.1); the related initial conditions can be transformed into (3.2). On this subject, see $\S 6$.

4. Scales of Banach algebras. There is one kind of scales of Banach spaces $X_{s}$ in which there are plenty of analytic mappings, and these are the scales of Banach algebras (each $X_{s}$ is a Banach algebra and if $s^{\prime} \leqq s, X_{s}$ is a subalgebra of $\left.X_{s^{\prime}}\right)$. In this section, we introduce a number of scales of Banach algebras in relation to which Cauchy problems of the kind of (1.10) or of (3.1)-(3.2) might be of some interest.

EXAMPLE 4.1. For each $s, 0 \leqq s \leqq 1$, let $K(s)$ be a compact subset of $C^{N}$, equal to the closure of its interior, assumed to be connected. If $s^{\prime}<s, K\left(s^{\prime}\right)$ is contained in the interior of $K(s)$ and, for some constant $c>0$,

(4.1) the distance from $K\left(s^{\prime}\right)$ to the boundary of $K(s)$ is $\geqq c\left(s-s^{\prime}\right)$.

Let then $X_{s}$ be the Banach space of continuous functions in $K(s)$ which are holomorphic in the interior of $K(s)$, equipped with the maximum norm. The restriction of functions from $K(s)$ to $K\left(s^{\prime}\right)\left(s^{\prime} \leqq s\right)$ defines a linear injection of $X_{s}$ into $X_{s^{\prime}}$ with norm $\leqq 1$; thus the $X_{s}$ form a scale of Banach spaces. Moreover, each one of them is a Banach algebra. Note also the following

Proposition 4.1. The partial differentiations $\partial / \partial z_{j}(j=1, \ldots, N)$ define bounded linear operators of $X_{s}$ into $X_{s^{\prime}}\left(0 \leqq s^{\prime}<s \leqq 1\right)$ with norm $\leqq c^{-1}\left(s-s^{\prime}\right)^{-1}$.

This is a straightfoward consequence of Cauchy's inequalities and of (4.1).

EXAMPLE 4.2. Let be given a sequence of numbers $\left\{c_{p}\right\}$ depending on the $N$ tuples $p=\left(p_{1}, \ldots, p_{N}\right)$ of $N$ integers $\geqq 0$, and satisfying for all $p$ 's,

$$
\sum_{q_{1}=0}^{p} \ldots \sum_{q_{N}=0}^{p_{N}}\left(\begin{array}{l}
p \\
q
\end{array}\right) c_{p-q} c_{q} \leqq c_{p} .
$$

Let $\Omega$ be an open subset of $R^{N}$. We denote by $\mathscr{C}^{\infty}\left(\Omega ;\left\{c_{p}\right\} ; M\right)(M$ : some number $>0$ ) the space of complex-valued $\mathscr{C}^{\infty}$ functions $\varphi$ in $\Omega$ such that

$$
\sup _{x, p}\left\{\frac{M^{|p|}}{c_{p}}\left|\varphi^{(p)}(x)\right|\right\}
$$

is finite. We may take (4.3) as the norm of $\varphi$ in $\mathscr{C}^{\infty}\left(\Omega ;\left\{c_{p}\right\} ; M\right)$ which then becomes a Banach space and, in fact, a Banach algebra-by virtue of (4.2).

Let then $M_{0}, M_{1}$ be two numbers such that $0<M_{0}<M_{1}$ and define $M(s)$ by $M(s)^{-1}=(1-s) M_{0}^{-1}+s M_{1}^{-1}$; we may then take as space $X_{s}$ the Banach algebra $\mathscr{C}^{\infty}\left(\Omega ;\left\{c_{p}\right\} ; M(s)\right)$. 
An important particular case of Example 4.2 is obtained when we choose $c_{k}=\varepsilon\left(k_{1}^{\prime} \cdots k_{N}^{\prime}\right)^{-2}(k !)^{d}$, where $d$ is $\geqq 1, \varepsilon>0$ is to be chosen below (so as to have (4.2)) and where we have written $k_{j}^{\prime}=\sup \left(1, k_{j}\right), j=1, \ldots, N$. We have:

$$
\begin{aligned}
\sum_{q_{1}=0}^{p_{1}} \ldots \sum_{q_{N}=0}^{p_{N}}\left(\begin{array}{l}
p \\
q
\end{array}\right) c_{p-q} c_{q} / c_{p} & \leqq \varepsilon \sum_{q_{1}} \cdots \sum_{q_{N}}\left(\begin{array}{l}
p \\
q
\end{array}\right)^{1-d} \prod_{j=1}^{N} \frac{p_{j}^{2}}{q_{j}^{2}\left(p_{j}-q_{j}\right)^{2}} \\
& \leqq \varepsilon\left(2+\sup _{p} \sum_{q=1}^{p-1} \frac{p^{2}}{q^{2}(p-q)^{2}}\right)^{N},
\end{aligned}
$$

where the prime in the product of the middle member indicates that any factor where the denominator vanishes must be taken to be one. We apply Lemma 2.1 and see that (4.2) is satisfied provided that we take $\varepsilon \leqq(2+4 S)^{-N}$. Observe that a change of the number $\varepsilon$ changes the norm in the space $\mathscr{C}^{\infty}\left(\Omega ;\left\{c_{p}\right\} ; M(s)\right)$ but not the space itself, let us for instance take $\varepsilon=(2+4 S)^{-N}$ and then denote by $G_{d}(\Omega ; M(s))$ the Banach algebra $\mathscr{C}^{\infty}\left(\Omega ;\left\{c_{p}\right\} ; M(s)\right)$.

Proposition 4.2. The partial differentiations $\partial / \partial x_{j}(j=1, \ldots, N)$ define bounded linear operators $G_{d}(\Omega ; M(s)) \rightarrow G_{d}\left(\Omega ; M\left(s^{\prime}\right)\right)\left(0 \leqq s^{\prime}<s \leqq 1\right)$ with norm $\leqq C\left(s-s^{\prime}\right)^{-d}$ where $C$ depends on $d, M_{1}-M_{0}, M_{0}^{-1}$.

Proof. It suffices to look at the case $N=1$. We have:

$$
\begin{aligned}
\left\|\varphi^{\prime}\right\|_{s^{\prime}} & =\sup _{x, p}\left\{\frac{M\left(s^{\prime}\right)^{p}}{\varepsilon p^{\prime 2}(p !)^{d}}\left|\varphi^{(p+1)}(x)\right|\right\} \\
& =\frac{1}{M\left(s^{\prime}\right)} \sup _{x, p}\left\{\left(\frac{p+1}{p^{\prime}}\right)^{2}\left[\frac{M\left(s^{\prime}\right)}{M(s)}\right]^{p+1}\left[\frac{M(s)^{p+1}}{(p+1)^{2}} \frac{\varphi^{(p+1)}(x)}{[(p+1) !]^{d}}\right](p+1)^{d}\right\} \\
& \leqq \frac{4}{M\left(s^{\prime}\right)}\|\varphi\|_{s} \sup _{p>0} p^{d}\left[\frac{M\left(s^{\prime}\right)}{M(s)}\right]^{p} \\
& \leqq \frac{4}{M\left(s^{\prime}\right)}(d / e)^{d} \frac{1}{\left[\log M(s)-\log M\left(s^{\prime}\right)\right]^{d}}\|\varphi\|_{s} .
\end{aligned}
$$

It is easy to check that $\log M(s)-\log M\left(s^{\prime}\right) \geqq C_{0}\left(s-s^{\prime}\right)$, where $C_{0}$ depends only on $\left(M_{1}-M_{0}\right) / M_{0} ;$ since $M\left(s^{\prime}\right) \geqq M_{0}$, the result follows at once.

EXAMPLE 4.3. Let $h$ be a real-valued continuous subadditive function in $R^{N}$. We take as space $X_{s}(0 \leqq s \leqq 1)$ the space of measurable functions $f$ in $R^{N}$ such that $e^{s h(\xi)} f(\xi) \in L^{1}$. In this case, $X_{s}$ is a Banach algebra for the convolution product, since

$$
\left|e^{\operatorname{sh}(\xi)} \int f(\xi-\eta) g(\eta) d \eta\right| \leqq e^{s[h(\xi)-h(\xi-\eta)-h(\eta)]} \int e^{\operatorname{sh}(\xi-\eta)}|f(\xi-\eta)| e^{s h(\eta)}|g(\eta)| d \eta
$$

and since $h(\xi) \leqq h(\xi-\eta)+h(\eta)$.

Suppose now $h \geqq 0$ and let $g$ be a measurable complex function in $R^{N}$ such that, for some numbers $C, a>0$,

(4.4) for every $\xi$ in $R^{N},|g(\xi)| \leqq C h(\xi)^{a}$. 
Proposition 4.3. Let $X_{s}$ be defined as in Example 4.3 and suppose that (4.4) holds. Then, if $0 \leqq s^{\prime}<s \leqq 1$, multiplication by $g$ defines a bounded linear operator $X_{s} \rightarrow X_{s^{\prime}}$ with norm $\leqq C(a / e)^{a}\left(s-s^{\prime}\right)^{-a}$.

Proof. It suffices to compute the maximum of $C h^{a} e^{-\left(s-s^{\prime}\right) h}$ for $h>0$.

EXAMPLE 4.4. This is the "Fourier transform" of Example 4.3. Let $\mathscr{S} \cap$ Exp denote the space of entire functions of exponential type on $C^{N}$ whose restrictions to $R^{N}$ decrease faster at infinity than any power of $1 /|x|$. The Fourier transforms of such functions are exactly the $\mathscr{C}^{\infty}$ functions in $R^{N}$ having compact support. We complete $\mathscr{S} \cap$ Exp for the norm

$$
\int e^{s h(\xi)}|\hat{u}(\xi)| d \xi
$$

here, $\hat{u}$ denotes the Fourier transform of $u$ :

$$
\hat{u}(\xi)=\int e^{-i\langle x, \xi\rangle} u(x) d x .
$$

Also, we should say that $h$ is a subadditive function in $R^{N} ; h \geqq 0$. The completion of $\mathscr{S} \cap \operatorname{Exp}$ for the norm (4.5) will be denoted by $K_{1}^{\text {sh }}$ (cf. [T1]). Since each space $X_{s}$, in Example 4.3, is a convolution Banach algebra, each $K_{1}^{s h}$ is a multiplication Banach algebra.

Let the measurable function $g$ satisfy (4.4) and set

$$
g(D) u(x)=(2 \pi)^{-n} \int e^{i\langle x, \xi\rangle} g(\xi) \hat{u}(\xi) d \xi
$$

We derive from Proposition 4.2

Proposition 4.3' $g(D)$ is a bounded linear operator $K_{1}^{s h} \rightarrow K_{1}^{s^{\prime} h}$ with norm $\leqq C(a / e)^{a}\left(s-s^{\prime}\right)^{-a}\left(0 \leqq s^{\prime}<s \leqq 1\right)$.

An important particular case of Example 4.4 is obtained with

(4.6) $h(\xi)=|\xi|^{1 / d}$,

where $d$ is any number $\geqq 1$. Then the elements of $K_{1}^{\text {sh }}$ can be identified with $\mathscr{C}^{\infty}$ functions in $R^{N}\left({ }^{2}\right)$ which satisfy a Gevrey condition of order $d$ (on this subject, cf. [T1, §14]). When $d=1$, these functions are analytic in $R^{N}$.

EXAMPLE 4.5. We consider a one-parameter family of compact sets $K(s)$ as in Example 4.1 except that this time, they are subsets of the Riemann sphere and not of $C^{N}$. We assume furthermore that the point at infinity $\infty$ belongs to the interior of every one of the sets $K(s)$. We denote by $Y_{s}$ the space of continuous functions in $K(s)$, holomorphic in the interior, vanishing at $\infty$. We equip $Y_{s}$ with

(2) This will be true whenever $(\log |\xi|) / h(\xi) \rightarrow 0$ as $|\xi| \rightarrow+\infty$-as can be checked at once. 
the maximum norm, which turns it into a Banach space, in fact a Banach algebra for the ordinary multiplication of functions. The "natural" injection of $Y_{s}$ into $Y_{s^{\prime}}$ when $s^{\prime} \leqq s$ is defined by the restriction of functions from $K(s)$ to $K\left(s^{\prime}\right)$.

Let $\Omega(s)$ be the complement of $K(s)$. We shall assume that $\Omega(s)$ is a bounded nonempty open subset of the complex plane (identified, as always, to the complement in the Riemann sphere of the point at infinity). Note that since $K(s)$ is connected, $\Omega(s)$ is simply connected. Let $\gamma$ be any simple rectifiable curve, contained in the interior of $K(s)$ and going around (counterclockwise) $\mathrm{cl}(\Omega(s)$ ); suppose furthermore that $\infty$ does not lie on $\gamma$. Given any $\varphi$ in $Y_{s}$,

$$
h \mapsto \int_{\gamma} h(z) \varphi(z) d z
$$

defines a continuous linear functional on the space of entire functions in $C^{1}$ (the latter is equipped with the topology of uniform convergence on the compact subsets of $C^{1}$ ). In other words, (4.7) defines an analytic functional, $M_{\varphi}$, in the complex plane. Since the cycle $\gamma$ can be taken arbitrarily near the boundary of $K(s)$, this boundary, or if one prefers, the closure $\mathrm{cl}(\Omega(s))$, carries the analytic functional $M_{\varphi}$. In particular we observe that (4.7) is defined not only for entire functions $h$ but for any holomorphic function in a neighborhood of $\operatorname{cl}(\Omega(s)$ ). By using this remark and taking the point $\zeta$ in the interior of $K(s)$, we may reconstruct $\varphi(\zeta)$ out of $M_{\varphi}$ by the formula

$$
\varphi(\zeta)=\frac{1}{2 i \pi}\left\langle M_{\varphi}, \frac{1}{\zeta-z}\right\rangle
$$

where $\langle$,$\rangle is the bracket of the duality between holomorphic functions and$ analytic functionals (in the variable $z$ ). If $\varphi$ and $M_{\varphi}$ are related by (4.8), $\varphi$ is called the Cauchy representation of $M_{\varphi}$. We write then $\varphi=\Phi M_{\varphi}$. On these and related subsets, see e.g. [T1, $\$ 17,18,19]$. Let us only mention that we have

$$
\Phi\{(d / d z) M\}=(d / d \zeta) \Phi M,
$$

(the derivative of an analytic functional is defined by the standard "integration by parts" formula $\left\langle M^{\prime}, h\right\rangle=-\left\langle M, h^{\prime}\right\rangle$ ).

When $\varphi$ ranges over $Y_{s}$ the analytic functionals $M_{\varphi}$ form a linear space $X_{s}$. Since $\varphi \mapsto M_{\varphi}$ is a linear bijection (whose inverse is $\Phi$ ), we may transfer the whole structure of $Y_{s}$ onto $X_{s}$. This turns $X_{s}$ into a Banach algebra. Observe that if $s^{\prime} \leqq s$, the analytic functionals belonging to $X_{s}$ are carried by a smaller compact set, namely $\mathrm{cl}(\Omega(s))$, than the one, $\mathrm{cl}\left(\Omega\left(s^{\prime}\right)\right)$, carrying arbitrary elements of $X_{s^{\prime}}$. The "natural" injection $X_{s} \rightarrow X_{s^{\prime}}$ is an "extension" mapping.

Analogous definitions can be introduced in the case of $N$ variables, by taking, as starting point, compact subsets of the product of $N$ Riemann spheres of the kind $K_{1}(s) \times \cdots \times K_{N}(s)$, where the $K_{f}(s)$ are similar to the sets $K(s)$ considered above. The analogue in $C^{N}$ of the open sets $\Omega(s)$ will be products $\Omega_{1}(s) \times \cdots$ 
$\times \Omega_{N}(s)$, where $\Omega_{j}(s)$ is the complement of $K_{j}(s)$ in the Riemann sphere. The Cauchy representation is then defined by

$$
\Phi M(\zeta)=(2 i \pi)^{-n}\left\langle M, \prod_{j=1}^{N}(\zeta-z)^{-1}\right\rangle .
$$

5. Examples of applications (I). By $E$ we denote a complex Banach space. Let us then consider a first order PDE of the kind:

$$
\partial u / \partial t=f\left(u, \partial u / \partial z_{1}, \ldots, \partial u / \partial z_{N}, z_{1}, \ldots, z_{N}, t\right),
$$

where $z=\left(z_{1}, \ldots, z_{N}\right)$ varies in some open subset $\Omega$ of $C^{N}$. The function $f$ is assumed to be analytic with respect to all its arguments, in an open subset of $E^{N+1} \times C^{N+1}$ of the form

$$
U_{0} \times U_{1} \times \cdots \times U_{N} \times \Omega \times D_{\eta}
$$

where the $U_{j}$ are open balls in $E$ and $D_{\eta}$ is the open disk $|t|<\eta$ in $C^{1}$. We look for a solution $u$ of (5.1) satisfying the initial condition:

$$
\left.u\right|_{t=0}=u_{0}(z)
$$

where $u_{0}$ is a holomorphic mapping of $\Omega$ into $U_{0}$ such that $\left(\partial / \partial z_{j}\right) u_{0}$ maps $\Omega$ into

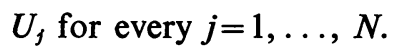

For each $j$, we set $v_{j}=\left(\partial / \partial z_{j}\right) u$ whence $(\partial / \partial t) v_{j}=\left(\partial / \partial z_{j}\right)(\partial / \partial t) u$, or

$$
(\partial / \partial t) v_{j}=\left(\partial / \partial z_{j}\right) f\left(u, v_{1}, \ldots, v_{N}, z_{1}, \ldots, z_{N}, t\right), \quad j=1, \ldots, N,
$$

which we adjoin to (5.1) rewritten in the form

$$
(\partial / \partial t) u=f\left(u, v_{1}, \ldots, v_{N}, z_{1}, \ldots, z_{N}, t\right) .
$$

The initial conditions are now, in addition to (5.2),

$$
\left.v_{j}\right|_{t=0}=\left(\partial / \partial z_{j}\right) u_{0}(z), \quad j=1, \ldots, N .
$$

Let now $K(s)$ be a one-parameter family of compact subsets of $\Omega$, exactly like the one considered in Example 4.1. In particular, we suppose that (4.1) holds. Let then $X_{s}$ be the Banach space consisting of the $(N+1)$-tuples $\left(u, v_{1}, \ldots, v_{N}\right)$ which are continuous in $K(s)$ and holomorphic in the interior of $K(s)$, equipped with, say, the sum of the maximum norms on each component $u, v_{1}, \ldots, v_{N}$. In virtue of the properties of the $K(s)$ listed in Example 4.1 the $X_{s}$ form a Banach space scale; Proposition 4.1 is valid. The image of $K(1)$, the largest of the compact sets $K(s)$, under $u_{0}$ (resp. $\left.\partial u_{0} / \partial z_{j}, j=1, \ldots, N\right)$ is a compact subset of $U_{0}$ (resp. of $U_{j}$ ). It follows from this that there is a number $r>0$ such that if $w=\left(w_{0}, w_{1}, \ldots, w_{N}\right)$ in $X_{s}$ satisfies

$$
\left\|w_{0}(z)-u_{0}(z)\right\|_{E} \leqq r, \quad\left\|w_{j}(z)-\left(\partial u_{0} / \partial z_{j}\right)(z)\right\|_{E} \leqq r
$$

for all $z$ in $K(s)$, then necessarily $w_{f}(z) \in U_{f}$ for all $z$ in $K(s)$ and for every $j=0,1$, $\ldots, N$. 
We may take the Taylor expansion of $f(w, z, t)$ about

$$
w=\left(u_{0}(z),\left(\partial u_{0} / \partial z_{1}\right)(z), \ldots,\left(\partial u_{1} / \partial z_{N}\right)(z)\right) \text { and } t=0,
$$

which we write in the form

$$
f(w, z, t)=\sum_{p, q} \hat{f}_{p, q}\left(w_{0}-u_{0}(z), w_{1}-\frac{\partial u_{0}}{\partial z_{1}}(z), \ldots, w_{N}-\frac{\partial u_{N}}{\partial z_{N}}(z) ; z\right) t^{-q},
$$

where $p=\left(p_{0}, p_{1}, \ldots, p_{N}\right)$ and $q=0,1, \ldots$ We have

$$
\hat{f}_{p, q}\left(w_{0}, w_{1}, \ldots, w_{N} ; z\right)=f_{p, q}\left(w_{0}^{1}, \ldots, w_{0}^{p_{0}} ; \ldots ; w_{N}^{1}, \ldots, w_{N}^{p_{N}} ; z\right),
$$

where the right-hand side is a multilinear function of the $w_{j}^{i}(j=0,1, \ldots, N$, $1 \leqq i \leqq p_{j}$ ) valued in $E$ and depending continuously on $z \in K(1)$, holomorphic with respect to $z$ in the interior of $K(1)$ (as a matter of fact, in view of our hypotheses on $u_{0}$, this multilinear mapping depends holomorphically on $z$ in a neighborhood of $K(1)$ ). From this it follows at once that, given any $s, 0 \leqq s \leqq 1$,

$$
\left(w_{0}^{1}, \ldots, w_{0}^{p_{0}}, \ldots, w_{N}^{1}, \ldots, w_{N}^{p_{N}}\right) \mapsto f_{p, q}\left(w_{0}^{1}, \ldots, w_{0}^{p_{0}} ; \ldots ; w_{N}^{1}, \ldots, w_{N}^{p_{N}} ; \ldots\right)
$$

is a multilinear mapping (which we shall denote by $F_{p, q}^{0}$ )

$$
X_{s}^{p_{0}} \times \cdots \times X_{s}^{p_{N}} \rightarrow X_{s} \text {. }
$$

It is not difficult to check that for some constant $C_{0}>0$,

$$
\left\|F_{p, q}^{0}\right\|_{s, s^{\prime}} \leqq C_{0} r^{-|p|} \eta^{-q} \text {. }
$$

Now, given any element $w$ in $X_{s}$ we may set

$$
F^{0}(w, t)=\sum_{p, q} \hat{F}_{p, q}^{0}\left(w_{0}-u_{0}, w_{1}-\frac{\partial u_{1}}{\partial z_{1}}, \ldots, w_{N}-\frac{\partial u_{N}}{\partial z_{N}}\right) t^{q}
$$

and viewing each $\partial / \partial z_{j}$ as a bounded linear operator $X_{s} \rightarrow X_{s^{\prime}}\left(s^{\prime}<s\right)$, we may set $F^{j}(w, t)=\left(\partial / \partial z_{j}\right) F^{0}(w, t)$ and $F(w, t)=\left(F^{0}(w, t), \ldots, F^{N}(w, t)\right)$. It is practically evident, in view of Proposition 4.1, that $F$ satisfies the hypotheses of Theorem 1.1. The conclusions therefore are valid and provide a version of the (nonlinear) Cauchy-Kovalevska theorem.

The Cauchy problem (5.1)-(5.2) can be given a meaning within the framework of Example 4.5. Let us assume $N=1$, for the sake of simplicity. In this case the sets $K(s)$ are not any more contained in $\Omega$, but the closures of their complements $\Omega(s)$ are. Here again Theorem 1.1 yields an existence-and-uniqueness result. But we must impose some restrictions upon the right-hand side in (5.1). Indeed, $f(u, \partial u / \partial z, z, t)$ must make sense when $u$ is an analytic functional belonging to a space $X_{s}$ introduced in Example 4.5. This can be achieved either by setting (as a definition) $f(u, \partial u / \partial z, z, t)=f(\Phi u,(\partial / \partial z)(\Phi u), z, t)\left({ }^{3}\right)$, or else by using the Banach algebra structure of $X_{s}$ (and also its structure of module over the space of holomorphic functions in $\Omega$ ).

(3) $\Phi$ denotes the Cauchy representation (see (4.8)). 
6. Examples of applications (II). We begin by describing, in rather vague terms, a quite general situation. We consider an equation of the type

$$
\partial^{m} u / \partial t^{m}=F\left(A^{0} u, A^{1} \partial u / \partial t, \ldots, A^{m-1} \partial^{m-1} u / \partial t^{m-1}, t\right) .
$$

Each $A^{j}$ is a linear operator acting in a certain way (to be made precise later) on the functions $u$ under consideration. It may depend on $t$; it may be of the form $A^{j} u=\left(A_{1}^{j} u, \ldots, A_{r}^{j} u\right)$. To (6.1) we adjoin the standard Cauchy conditions,

$$
\partial^{j} u /\left.\partial t^{j}\right|_{t=0}=u_{0}^{j}, \quad j=0,1, \ldots, m-1 .
$$

Under suitable assumptions about $F$ and the $A^{j}$ this Cauchy problem can be reduced to the problems considered in $\S 3$. First of all, we set

$$
u^{j}=\partial^{j} u / \partial t^{j}, \quad 0 \leqq j<m,
$$

which, in conjunction with (6.1), yields the new system:

$$
\begin{gathered}
\partial u^{j} / \partial t=u^{j+1}, \quad j=0, \ldots, m-2, \\
\partial u^{m-1} / \partial t=F\left(A^{0} u^{0}, A^{1} u^{1}, \ldots, A^{m-1} u^{m-1}, t\right) .
\end{gathered}
$$

We must perform one more reduction, on (6.4). Let us set $v^{j}=A^{j} u^{j}, 0 \leqq j<m$. This replaces $(6.4)$ by

$$
\partial u^{m-1} / \partial t=F\left(v^{0}, v^{1}, \ldots, v^{m-1}, t\right)
$$

and we must adjoin to (6.3) and (6.5) the equations

$$
\begin{gathered}
\partial v^{j} / \partial t=A^{j} u^{j}+A^{j} u^{j+1}, \quad j=0, \ldots, m-2, \\
\partial v^{m-1} / \partial t=A^{m-1} u^{m-1}+A^{m-1} F\left(v^{0}, v^{1}, \ldots, v^{m-1}, t\right),
\end{gathered}
$$

where $A^{j}=(d / d t) A^{j}$. Equations (6.3), (6.5), (6.6), (6.7) form a system of $2 m$ equations in $2 m$ unknowns. The initial conditions are:

$$
\left.u^{j}\right|_{t=0}=u_{0}^{j},\left.\quad v^{j}\right|_{t=0}=A^{j}(0) u_{0}^{j}, \quad j=0, \ldots, m-1,
$$

where $A^{j}(0)=A^{j}$ at $t=0$. We shall assume that each $A^{j}$ acts from a Banach space scale $X_{s}^{j}$ into another such scale, $Y_{s}^{j}$. More precisely, if $0 \leqq s^{\prime}<s \leqq 1, A^{j}$ induces a bounded linear operator, depending holomorphically on $t$ in $D_{\eta}$, from $X_{s}^{j}$ into $Y_{s^{\prime}}^{j}$. We will have the following bound on the norm of this operator:

$$
\left\|A^{j}\right\|_{s, s^{\prime}} \leqq C\left(s-s^{\prime}\right)^{j-m} \quad(j=0, \ldots, m-1) .
$$

Here $C$ is a constant, independent of all the data. As for the function $F$, it will have to be valued in $X_{s}^{0}$ and to be defined and analytic in an appropriate open subset of $Y_{s}^{0} \times Y_{s}^{1} \times \cdots \times Y_{s}^{m-1}$, whatever $s, 0 \leqq s \leqq 1$. With respect to $t$, it will be analytic in $D_{\eta}$.

Let us define $2 m$ intègers $d^{i}(i=1, \ldots, 2 m)$ as follows:

$$
d^{i}=0 \quad \text { if } i \leqq m, \quad d^{i}=2 m+1-i \quad \text { if } m<i \leqq 2 m .
$$


In virtue of (6.9) this fits with the definition of the $d^{i}$ 's in $\S 3$, specifically in (3.3), when we apply it to the system (6.3), (6.5), (6.6), (6.7). Next we introduce the number $\theta_{j}^{i}$ as in (3.4). Observe that

$$
\begin{array}{rll}
\text { if } i<m, & \theta_{j}^{i}=1 & \text { if } j=i+1, \text { zero otherwise; } \\
& \theta_{j}^{m}=1 & \text { when } j=m+1, \ldots, 2 m, \text { zero otherwise; } \\
\text { if } m<i<2 m, \quad \theta_{j}^{i}=1 & \text { if } j=i-m \text { or } j=i-m-1, \text { zero otherwise; } \\
\theta_{j}^{2 m}=1 & \text { when } j=m, m+1, \ldots, 2 m, \text { and zero otherwise. }
\end{array}
$$

Under these circumstances it is not difficult to check that (3.5) holds (where $2 \mathrm{~m}$ must now be substituted for $m$ ). It suffices to show that the degree of (3.4) with respect to $\lambda$, when we put $\mu=c \lambda$ with $c$ fixed but arbitrary, is $\leqq 2 m$. Since the $c_{j}^{i}$ are arbitrary, it suffices to take $\mu=\lambda$. We leave the details to the student.

We are thus in the situation where to apply Theorem 3.1. We discuss now rapidly two particular cases.

EXAMPLE 6.1. The basic scale of Banach spaces will be here the spaces $G_{d}(\Omega ; M(s))$ considered in Proposition 4.2. We take the $A^{j}$ to be linear partial differential operators in the $x$-variables with smooth coefficients depending on $x$ and $t$. The degree of smoothness of these coefficients with respect to $x$ must be such that $A^{j}$ operates on all the $G_{d}(\Omega ; M(s))$ for $0 \leqq s \leqq 1 ; A^{j}$ must furthermore be a holomorphic function of $t$ in $D_{\eta}$. We may therefore take the entries of the coefficients (in general, these are matrices) to be holomorphic functions of $t$ in $D_{\eta}$ valued in the Banach algebra $G_{d}(\Omega ; M(1))$. Let $\omega^{j}$ be the order of the differential operator $A^{j}$. If we combine Proposition 4.2 with the requirement (6.9) we find the "standard" condition:

$$
j+\omega^{j} d \leqq m, \quad j=0, \ldots, m-1 .
$$

EXAMPLE 6.2. The basic scale of Banach spaces will now be the spaces $K_{1}^{\text {sh }}$ introduced in Example 4.4. We may take the $A^{j}$ to be convolution operators of the type $g(D)$ considered in Proposition 4.3'. Let us say that the order of the operator $g(D)$ is $\leqq a$ if (4.4) holds. Then (6.9) requires that the order of $A^{j}$ be $\leqq m-j$.

For instance, if $h(\xi)=|\xi|^{1 / d}(d \geqq 1)$, this means that the "symbol" $a^{j}(\xi)$ of $A^{j}$ must satisfy $\left\|a^{j}(\xi)\right\|^{d} \leqq C|\xi|^{m-j}$. The analogy with Example 6.1 is self-evident.

In the context of the spaces $K_{1}^{s h}$ we may take the $A^{j}$ to be of a far more complex type than the convolution operators just considered. We may take them to be similar to "pseudodifferential" operators, given by

$$
A^{j} u(x)=(2 \pi)^{-n} \int e^{i\langle x, \xi\rangle} a^{j}(x, \xi) \hat{u}(\xi) d \xi .
$$

Of course, the class of symbols $a^{j}(x, \xi)$ for which this would work (that is, such that $A^{j}$ would operate appropriately on the $K_{1}^{s h}$ ) must be circumscribed. Observe that we can obtain a large quantity of multipliers on the $K_{1}^{\text {sh }}$ by taking the elements 
themselves of this Banach algebra. This in turn yields a large class of admissible symbols, the finite sums of products of a convolver and a multiplier. From there on we may proceed by completion (we may also proceed directly by imposing bounds on the Fourier transform of the symbol $a^{j}(x, \xi)$ with respect to $x$ ).

The function $F\left(v^{0}, v^{1}, \ldots, v^{m-1}, t\right)$ entering in (6.1) might be defined, in the applications, by functions $f\left(v^{0}, v^{1}, \ldots, v^{m-1}, x_{1}, \ldots, x_{N}, t\right)$, analytic with respect to $t$ and with respect to the $v^{j^{\prime}}$ (assumed now to vary in certain open subsets of spaces $C^{r_{j}}$ or even of Banach spaces $E$ like in $\S 5$ ) and having a certain type of smoothness with respect to $x$, adapted to the scales of Banach spaces (or Banach algebras) $X_{s}$ one is dealing with. A large degree of freedom is available here in choosing the kind of functions $f$ to which one would apply the general results.

\section{REFERENCES}

[O1] L. V. Ovsjannikov, Singular operators in Banach spaces scales, Dokl. Akad. Nauk SSSR 163 (1965), 819-822 = Soviet Math. Dokl. 6 (1965), 1025-1028. MR 32 \#8164.

[S-T1] S. Steinberg and F. Treves, Pseudo-Fokker Planck equations and hyperdifferential operators, (to appear).

[T1] F. Treves, On the theory of linear partial differential equations with analytic coefficients, Trans. Amer. Math. Soc. 137 (1969), 1-20.

[T2] - Ovcyannikov theorem and hyperdifferential operators, Instituto de Matematica Pura e Aplicada, Rio de Janeiro, 1968.

PURdue University,

LAFAYETTE, INDIANA 47907 